Determinantes de la oferta primaria de ganado vacuno para ceba en el departamento de Córdoba, Colombia

Omar Enrique Castillo Núñez 
Determinantes de la oferta primaria de ganado vacuno para ceba en el departamento de Córdoba, Colombia

Resumen: En este articulo, se identifican determinantes de la oferta primaria de ganado vacuno para cebar en el departamento de Córdoba, Colombia, durante el período 2007-2018. Para ello se utiliza un modelo econométrico lineal autorregresivo con retardos distribuidos que permite, mediante la prueba limite, establecer las relaciones de largo y de corto plazo entre las variables. Los resultados empiricos evidencian que los ganaderos responden a estímulos de mercado, como precios y costos de producción; son adversos al riesgo-clima y al riesgo-precio; compiten por recursos productivos con un cultivo como el maí; la predominancia del sistema de producción del doble propósito y la presencia de comercializadores intermediarios que realizan ceba incompleta explican una relación directa entre el ciclo de la ceba final y la oferta primaria.

Palabras clave: economía agraria, microeconomía, análisis de series de tiempo, prueba limite, modelo corrección del error.

Clasificación JEL: B21, C22, Q11.

\section{Determinants of the Primary Supply of Cattle for Fattening in the Department of Córdoba, Colombia}

Abstract: Factors for the primary supply of livestock, for fattening are identified in the Department of Cordoba, Colombia, during 2007-2018. For this, a linear autoregressive econometric model with distributed delays is used, which allows by a limit proof, to establish long-and short-term relationships between the variables. The empirical results show that livestock traders react to market stimulus, such as prices and production costs; they are averse to weather risk and price risk; they compete for productive resources with a crop such as corn. Similarly, the predominance of the double purpose production system and the presence of intermediary traders who carry out incomplete fattening explains a direct relationship between the cycle of final fattening and primary supply.

Keywords: agrarian economics, microeconomics, time series analysis, test bound, error correction model.

https://doi.org/10.17533/udea.le.n96a343891

\section{(cc) BY-NC-SA}

Este artículo y sus anexos se distribuyen por la revista Lecturas de Economía bajo los términos de la Licencia Creative Commons Atribución-NoComercial-CompartirIgual 4.0. https://creativecommons.org/licenses/by-nc-sa/4.0/ 


\section{Déterminants de l'offre primaire en bovins d'engraissement dans le Départe- ment de Córdoba, en Colombie}

Résumé: Cet article identifie les déterminants de l'offre primaire de bétail à engraisser dans le département de Córdoba, en Colombie, au cours de la période 2007-2018. Pour ce faire, un modèle économétrique linéaire autorégressif avec des retards distribués est utilisé, permettant, grâce au test de limite, d'établir les relations à long terme et à court terme entre les variables. Les résultats empiriques montrent que les agriculteurs réagissent aux stimuli du marché, tels que les prix et les coûts de production; ils sont défavorables au risque-climat et au risque-prix; concurrencer les ressources productives avec une culture comme le maï; la prédominance du système de production à double objectif et la présence d'intermédiaires qui effectuent un engraissement incomplet, expliquent une relation directe entre le cycle de l'engraissement final et l'approvisionnement primaire.

Mots clés: économie agricole, microéconomie, analyse de séries chronologiques, test de limite, modèle de correction d'erreur.

Cómo citar / How to cite this item:

Castillo Núñez, O. E. (2022). Determinantes de la oferta primaria de ganado vacuno para ceba en el departamento de Córdoba, Colombia. Lecturas de Economía, 96, 279-314. https://doi.org/10.17533/udea.le.n96a343891 


\title{
Determinantes de la oferta primaria de ganado vacuno para ceba en el departamento de Córdoba, Colombia
}

\author{
Omar Enrique Castillo Núñez $\circledast^{a}$
}

-Introducción. -I. Revisión de literatura. -II. Modelo econométrico. -III. Discusión de resultados. -Conclusiones. -Referencias

Primera versión recibida el 20 de septiembre de 2020; versión final aceptada el 31 de mayo de 2021

\section{Introducción}

El departamento de Córdoba, Colombia, se caracteriza por ser productor de carne de ganado vacuno con técnicas de alimentación basada en pasturas naturales. En la encuesta nacional agropecuaria, ENA, del año 2018, de 975715 cabezas con orientación a la producción de carne en las unidades productivas existentes, el $49 \%$ estaban en etapa de ceba, $23 \%$, en cría y levante, y $28 \%$ en ceba completa (DANE, 2018a).

El modelo empresarial predominante de producción de carne no es homogéneo, desde el punto de vista de la provisión de los animales para cebar. Algunas unidades productivas integran la primera y la segunda etapa proveyéndoles el ternero de levante con la producción de la misma unidad productiva procedente de la etapa vaca-cría. Otras, lo compran en mercados organizados o en unidades productivas de cría para hacer ceba intermedia o completa. La principal fuente de provisión del ganado de levante de estas últimas han sido las subastas de ganado vacuno vivo. En promedio, según el Observatorio de Precios y Costos Agrarios del Noroccidente del Caribe Colombiano (OPCA, 2018), durante el período enero/2007-diciembre/2018, una proporción del 50 por ciento del ganado ofrecido y vendido en subastas comerciales de primera clase fueron animales machos y hembras de menos de 1 hasta 1,75 años, con peso físico medio entre 116 y 234 kilogramos.

a Omar Enrique Castillo Núnez: profesor titular de la Universidad de Córdoba, Facultad de Ciencias Agrícolas, Montería, Córdoba, Colombia. Dirección electrónica: ocastillo@correo.unicordoba.edu.co https:/ / orcid.org/0000-0002-8403-7729 
La indagación empírica sobre los factores relacionados con el ambiente físico y económico, que influyen en el comportamiento de los agentes, que proveen esta oferta primaria de ganado vacuno en pie para cebar ha sido poco explorada. ¿Responden los productores a las variaciones del precio del producto y al precio de otros relacionados? ¿Cuánto afecta el riesgo-precio? ¿cuánto afecta el riesgo-clima? Córdoba es el principal productor de maíz en Colombia, ¿Compite este cultivo con la producción de ganado vacuno? ¿Afecta el ciclo de la ceba la oferta del ganado de levante?

Este artículo da respuestas empíricas a estas preguntas y, en general, identifica algunos determinantes de la oferta de ganado vacuno macho y hembra de levante para ceba durante el período enero/2007-diciembre/2018. Para ello, se estima un modelo econométrico lineal de series de tiempo que los asocia con el fin de evaluar su efecto en la evolución de la oferta a largo y corto plazo.

Los resultados aportan evidencia empírica adicional acerca de la dinámica de la oferta de mercado de ganado para cebar en la subregión del Noroccidente del Caribe Colombiano de la cual hace parte el departamento de Córdoba. Proporciona elementos de análisis al sector público y a los gremios privados en el eslabón primario de la cadena cárnica acerca de los incentivos económicos a los que responden productores y comercializadores, el efecto del riesgo-precio y del riesgo-clima para afrontar situaciones indeseables en la economía ganadera regional. Luego de esta introducción, se presenta la revisión de literatura en el acápite uno; en el dos, el modelo econométrico que se estima; en el tres, se muestran y discuten resultados; en el cuarto, se esbozan las conclusiones

\section{Revisión de literatura}

La función de la oferta total agraria se refiere a la relación analítica existente entre la oferta de un producto agrario y los factores determinantes de la misma (Tomek \& Robinson, 2005) Los principales hallazgos encontrados sobre esta relación se señalan a continuación. 


\section{A. El precio del producto}

Bajo el supuesto de racionalidad y estructuras de mercado competitivas, la teoría económica dominante predice que si los otros factores que afectan la oferta permanecen constantes - y si el costo marginal de producción es creciente-, entre mayor sea el precio, mayor es la disposición del productor a ofrecer una cantidad mayor de producto. Los principales hallazgos empíricos de la literatura en este tema han sido resumidos por Mundlak (2001). En particular, destaca que: i) las elasticidades decrecen con el nivel de agregación: son más altas cuando se estiman para productos individuales que para el producto agregado; ii) cuando se introduce el producto retardado en la ecuación empírica de la oferta, mejora el ajuste y elimina la correlación serial. En la industria ganadera, Jarvis (1974) mostró que el envío de animales adultos a sacrificio tiene dos efectos: uno es incrementar la oferta de carne; el otro es reducir el tamaño del rebaño de cría.

La investigación nacional sobre oferta ganadera final destinada a sacrificio ha sido abordada por Jaramillo y Caicedo (1996), García (1983), Hertford y Nores (1982), DNP (1980), y Lorente (1978). Estos autores utilizan información anual y como variable dependiente la oferta interna final o de consumo - esto es, el sacrificio más las exportaciones—; en otras utilizan una medida relativa como lo es la tasa de extracción. Siguiendo a Jarvis (1974), se identificaron una relación positiva de largo plazo con el precio, y una relación inversa de corto plazo, la cual es expresión del ciclo ganadero de la ceba.

En general, desde el punto de vista de la metodología econométrica utilizada, sus resultados suponen que las series de tiempo de las variables utilizadas tienen propiedades estadísticas estacionarias y al combinarlas omiten que pueden estar cointegradas $^{1}$.

1 Desde Nelson y Plosser (1982) — quienes encontraron no estacionariedad en variables macroeconómicas de Estados Unidos - las pruebas de raíces unitarias son una práctica estándar. Y desde Engle y Granger (1987), las pruebas para contrastar la existencia de una combinación lineal estacionaria en series no estacionarias es también pan de cada día en la Econometría de series de tiempo. 
Castillo Núñez: Determinantes de la oferta primaria de ganado vacuno para ceba...

\section{B. Cambios en el precio de los insumos}

Teóricamente — todo lo demás constante—, a nivel del productor, el incremento del precio de un insumo implica un aumento del costo medio del mismo lo cual significa que el costo medio de producción aumenta, desplazándose la nueva curva de costo medio hacia arriba de la inicial. La curva de costo marginal se desplaza hacia atrás como expresión que la nueva relación precio del insumo/precio del producto ha aumentado, esto es, se ha encarecido el precio relativo del insumo lo cual reduce la cantidad usada del mismo y la cantidad de producto óptima, como lo indica la teoría microeconómica clásica (Hudson, 2007). Como la oferta de mercado es la suma de las ofertas individuales, la relación precio del insumo y oferta es inversa.

La relación precio del insumo/precio del producto ha sido importante en la determinación de la oferta agraria, especialmente en productos ganaderos (Castillo, 2015). En estos, los cambios en los costos de alimentación influyen sobre la ganancia económica y los cambios en el uso de alimentos influencian la cantidad del producto. Por lo tanto, para predecir la oferta, los analistas han puesto atención al uso de los alimentos como insumo.

En la estructura de costos de la ganadería el componente de manejo de praderas representa alrededor del $21 \%$ del costo total en ganadería de leche, $18 \%$ en carne y $13 \%$ en doble propósito (SIPSA, 2008). Perfetti et al. (2012) indicaron que la fertilización representa el $63 \%$ del valor de los insumos en el manejo de praderas en Córdoba, Cesar y Meta.

\section{Modificaciones en la rentabilidad de los productos competentes}

Productos competentes son aquellos que compiten por el uso de recursos productivos durante el ciclo de producción. La oferta de un producto competente disminuye si la rentabilidad del otro producto alternativo crece. La rentabilidad de este último aumenta si su precio aumenta más rápido que el del primero; o porque su costo de producción relativo disminuye más rápido que el otro (Tomek \& Robinson, 2005). Esto último podría provenir de un avance tecnológico que eleve la productividad de este con respecto al otro 
e incremente la rentabilidad relativa. Bajo racionalidad y mercados perfectos, una variación en la rentabilidad de un producto competente tiende a mantener una relación inversa con la oferta del otro.

\section{Variación del precio del coproducto}

La oferta de un producto agrario es determinada —en parte- por relaciones conjuntas. En la ganadería bovina, el sistema de doble propósito es producción conjunta, esto es, se obtienen varios productos utilizando los mismos insumos y factores de producción o, al menos, con factores e insumos comunes. La leche cruda es un coproducto de la carne de ganado vacuno pues son creados en el mismo proceso y al mismo tiempo. La teoría económica sugiere que el precio del coproducto influye sobre la oferta del otro producto en sentido directo. El aumento del precio de la leche representa un estímulo para los ganaderos que optaron por aumentar su inventario de vacas o mejorar la nutrición y la salud de las reses, lo cual se traduciría en aumento del número de terneros llevados al mercado ${ }^{2}$.

\section{E. Riesgo e incertidumbre}

En la producción agraria, estas se deben al hecho que se trabaja con seres vivos y depende de elementos incontrolables como el tiempo atmosférico y el clima (riesgo-clima). La variabilidad del precio es otro atributo estándar de las actividades agrarias (riesgo- precio) y está asociado a la inestabilidad de los mercados agrícolas por dos razones: los choques de oferta por cuestiones climáticas y la inelasticidad — precio de la oferta - y demanda primaria por productos agrícolas. Como resultado de ello, pueden variar con el tiempo haciéndolos difícil de predecir (Chavas et al., 2014).

La evidencia empírica en el examen del efecto del riesgo sobre la oferta agraria ha sido resumida por Chavas (2019), Chavas et al. (2010), Moschini y Hennessy (2001). Sugieren que las variables de riesgo son significativas en la

2 De 2007611 cabezas reportadas en la ENA del año 2018, DANE (2018a), la orientación productiva de las unidades de producción de la ganadería vacuna en Córdoba es: de doble propósito, 38,9\%; carne, 48,6\%\%; leche, $12,5 \%$. Tabla 61. 
Castillo Núñez: Determinantes de la oferta primaria de ganado vacuno para ceba...

explicación de las decisiones de producción agraria. Se espera que el productor adverso al riesgo produzca menos que el productor neutral al riesgo, ceteris paribus, y que ajuste el producto a las condiciones cambiantes del riesgo —esto es, disminuirá la producción cuando el riesgo es mayor-. Además, la inclusión del riesgo en los modelos econométricos de estimación de la función de oferta sugiere que el impacto del precio se vuelve más importante. Es decir, cuando se tiene en cuenta el riesgo, la oferta es más sensible al precio.

La técnica de producción ganadera predominante en la región bajo estudio es la del pastoreo, en la que el animal es alimentado con pastos naturales y forrajes. Bajo este sistema, el riesgo-clima tiene efectos importantes sobre el crecimiento de los ganados, las tasas de reproducción, las tasas de morbilidad y mortalidad, la oferta de alimentación, el contenido nutricional y la incidencia de pestes y enfermedades. Algunos hallazgos específicos encontrados en la literatura son:

(i) La precipitación pluvial afecta la distribución y cantidad de pasto, los déficits hídricos disminuyen la oferta de alimento, de modo que es necesario un período más largo de alimentación para obtener el mismo volumen de producción animal (Mader et al. 2009).

(ii) La ausencia o el exceso de la lluvia aumenta la incidencia de pestes y enfermedades, alterando la salud animal y la fecundidad (Gale et al. 2009).

(iii) Se ha observado que el incremento en la intensidad de las lluvias amplía el rango de degradación de suelos y pastizales (Howden, Crimp \& Stokes, 2008).

En lo regional, Bracamonte et al. (2018) evidencian el efecto del riegoclima en el largo y corto plazo, y riesgo-precio en el corto plazo en la ganadería del departamento de Sucre, Colombia. Manifestaciones de volatilidad o riesgoprecio, medida por la varianza condicional del precio semanal de ganado de levante en Montería, Córdoba, han sido reportadas por Castillo (2009). 


\section{F. Ciclo del ganado vacuno de ceba}

La oferta primaria de ganado vacuno de levante para cebar es afectada por el ciclo ganadero. La existencia del ciclo en ganadería de ceba ha sido abordada por Tonsor y Mitchell (2017), Mundlak y Huang (1996) e Yver (1972). Señalan que, cuando el precio de la carne de res aumenta, los productores piensan que tal incremento es permanente, reaccionan reteniendo más ganado hembra y, por lo tanto, reducen la oferta de carne en el corto plazo. La razón por la que actúan de esta manera es porque esperan que el incremento del precio persistirá en el futuro y la rentabilidad de largo plazo aumentará. Entre más altas sean en el largo plazo las expectativas de ganancia, más rápido incrementarán su capital y lo harán reteniendo más terneros para aumentar su stock de vacas y toros. Esta es la fase de retención. En ella disminuye el sacrificio, especialmente la proporción de hembras; al escasear el ganado para sacrificio se acentúa la demanda por ganado de levante para engorde, lo cual lo valoriza relativamente incrementando la oferta primaria por la vía del aumento del precio. Por el contrario, si la expectativa es de reducción del precio y de la rentabilidad ganadera, se produce una fase de liquidación. En esta aumenta la proporción sacrificada de hembras y decae el precio relativo del ganado de levante.

La presencia del ciclo en la ganadería en Colombia ha sido destacada por Escobar y Rojo (2014), Parra y Gómez, (2008), Pérez (2004), Jaramillo y Caicedo (1996), Lorente (1986), entre otros.

La respuesta de la oferta primaria al ciclo podría ser diferenciada. En un sistema de producción de solo carne, un productor-cebador directo bajo racionalidad se ajusta al mecanismo descrito. En un sistema de producción de doble propósito — como el predominante en la región_, la venta de leche podría alterar el mecanismo tradicional del ciclo. Un comercializador maximizador de ganancias inmediatas buscaría realizarlas independiente de las fases del ciclo.

\section{Modelo econométrico}

La contrastación de las predicciones de la teoría sobre los determinantes de la oferta con la realidad empírica ha seguido dos enfoques: i) el modelo 
Castillo Núñez: Determinantes de la oferta primaria de ganado vacuno para ceba...

de ajuste parcial nerloviano, (Nerlove \& Bessler, 2001), pero el producto deseado ni el precio esperado son observables; ii) el enfoque de la función de oferta, que deriva la oferta de la maximización de la función de ganancia (Mundlak, 2001) ${ }^{3}$.

La aplicación de cualquiera de estos enfoques es complicada en la zona de estudio. En el primero, los mercados de futuros en ganado son inexistentes y no pueden ser utilizados para el análisis; hay evidencia empírica —ademásde expectativas heterogéneas entre los productores (Chavas, 2000). En el segundo enfoque, la estructura detallada de datos que exige sobre precios de los insumos es un obstáculo real: algunos mercados de factores como el de la tierra, laboral y de crédito están ausentes o poseen estructuras de mercado imperfectas (Cano, 2016; González y Bonilla, 2016).

En consecuencia, se optó por utilizar el enfoque del modelo autorregresivo con retardos distribuidos, ARDL. Este tiene características deseables:

i) Se apoya en regresiones mínimo-cuadráticas estándar, que incluyen retardos tanto de la variable dependiente como de las variables explicativas relacionándolas con valores contemporáneos e históricos, lo cual da dinámica a las respuestas de las variables.

ii) Ha ganado popularidad en años recientes por las innovaciones metodológicas propuestas por Pesaran et al., (2001) y Pesaran y Shin (1999), en el caso del enfoque ARDL lineal; y por Shin et al., (2014) en el enfoque no lineal. Permite, por tanto, examinar relaciones lineales y no lineales de largo y de corto plazo entre variables económicas estacionarias, $I(0)$, y no estacionarias de orden $1, I(1)$.

iii) A diferencia de las pruebas de cointegración de Johansen, (1995), Park (1992), Phillips y Ouliaris (1990) o Engle-Granger (Engle \& Granger, 1987) que requieren que todas las variables del vector autorregresivo, $V A R$, sean $I(1)$ para determinar relaciones de corto y largo plazo entre las variables, este enfoque propone una prueba de cointegración que es

3 Se remite al lector a Haile et al. (2016) para una revisión de las críticas al primer enfoque, sobre todo a la cuestión de especificar cómo se forman las expectativas del precio; y al segundo sobre los requerimientos de información detallada de los precios de los insumos y la estimación simultánea de la demanda de insumos y la función de oferta del producto. 
robusta a si las variables de interés son $I(0), I(1)$ o una combinación de ambas. No es válido si se tienen variables integradas de orden $2, I(2)$.

Si $y_{t}$ es la variable dependiente y $x_{1}, \ldots, x_{k}$ son $k$ variables independientes o explicativas. Un modelo general $A R D L\left(p, q_{1}, \ldots, q_{k}\right)$ tiene la siguiente representación:

$$
y_{t}=a_{0}+a_{1} t+\sum_{i=1}^{p} \psi_{i} y_{t-i}+\sum_{j=1}^{k} \sum_{l_{j}=0}^{q_{j}} \beta_{j, l_{j}} x_{j, t-l_{j}}+\varepsilon_{t}
$$

$a_{0}, a_{1}, \psi_{i}$ y $\beta_{j, l}$ son, respectivamente, los coeficientes asociados con un intercepto, una tendencia lineal, los retardos de $y_{t} \mathrm{y}$ los retardos de los $k$ regresores $x_{j, t}$ para $j=1, \ldots, k$.

$p$ y $q$ representan el orden de los retardos de la variable dependiente y de las variables explicativas, respectivamente.

$\varepsilon_{t}$ representa las perturbaciones o innovaciones, idéntica e independiente distribuidas con media cero, varianza constante y no correlacionadas entre sí.

Considérese $L$ como el operador de retardo y $\psi(L)=1-\sum_{i=1}^{p} \psi_{i} L^{i}$ y $\beta_{j}(L)=1-\sum_{l_{j=1}}^{q_{j}} \beta_{j, l_{j}} L^{l j}$ como retardos polinomiales:

Entonces, la ecuación 1 puede ser reescrita como:

$$
\psi(L) y_{t}=a_{0}+a_{1} t+\sum_{j=1}^{k} \beta_{j}(L) x_{j, t}+\varepsilon_{t}
$$

A partir de esta formulación general, Pesaran et al. (2001) demostraron que la ecuación 1 se puede reducir a una representación como la siguiente.

$$
\begin{array}{r}
\Delta y_{t}=a_{0}+a_{1} t-\psi(1) E C_{t-1}+\left(\widetilde{\psi}^{*}(L) \Delta y_{t-1}+\sum_{j=1}^{k} \widetilde{\beta}_{j}(L) \Delta x_{j, t-1}\right) \\
+\sum_{j=1}^{k} \beta_{j}(L) \Delta x_{j, t}+\varepsilon_{t}
\end{array}
$$


Castillo Núñez: Determinantes de la oferta primaria de ganado vacuno para ceba...

En la ecuación (3) se tiene que:

$\psi(1)$ es un término constante o velocidad de ajuste hacia el equilibrio.

$E C_{t-1}$, es el término corrección del error o la ecuación cointegrante del conocido modelo. $\operatorname{VAR}(p): \Phi(L)\left(z_{t}-\mu-\gamma t\right)=\epsilon_{t}$ cuando $y_{t} \mathrm{y}$ $x_{1, t}, \ldots, x_{k, t}$ están cointegradas. $z_{t}=$ es el $(k+1)$ vector, $\left(y_{t}, x_{1, t}, \ldots, x_{k, t}\right)^{T}$.

$\mu, \gamma$ representan respectivamente $(k+1)$ vectores de los coeficientes de intercepto y tendencia desconocidos.

$\Phi(L)=I_{k+1}-\sum_{i=1}^{p} \Phi_{i} L^{i}$ es la matriz cuadrada $(k+1)$ de los retardos del polinomio.

La identidad de la ecuación 3 del enfoque ARDL con el enfoque de cointegración del modelo $\operatorname{VAR}(p)$ se hace evidente a través de la prueba límite (Test Bound). Esta es la prueba de significancia de los parámetros en la ecuación de cointegración del modelo corrección del error, que define la existencia de relaciones de largo plazo en niveles entre la variable dependiente y las explicativas.

La prueba límite es una $F$ estándar de significación conjunta o una prueba de Wald de la hipótesis nula, $H_{0}$, y alternativa, $H_{1}$ (Pesaran et al., 2001, pp. 9-11).

$$
\begin{array}{r}
H_{0}:\left(\psi(1) \cap\left\{\beta_{J}(1)_{j=1}^{k}\right\}\right)=0 \\
H_{1}:\left(\psi(1) \cap\left\{\beta_{J}(1)_{j=1}^{k}\right\}\right) \neq 0
\end{array}
$$

La expresión (4) es la hipótesis nula $H_{0}$ : no hay ninguna relación de largo plazo entre la variable dependiente y las explicativas del modelo. La hipótesis alternativa, $H_{1}$, es: sí existe esa relación.

Una vez que se calcula el estadístico $F$ se compara con dos valores críticos asintóticos correspondientes a dos casos polares de todas las variables siendo puramente $I(0)$ o $I(1)$. Si este valor calculado del estadístico $F$ está por debajo del valor crítico más bajo, se fracasa en rechazar la hipótesis nula y se concluye que no existe relación de largo plazo entre las variables, es decir, no están cointegradas. Lo contrario, cuanto el valor del estadístico está por 
encima del valor crítico más alto, se tiene evidencia para rechazar la hipótesis nula y aceptar que la cointegración es, en efecto, posible. Si el estadístico cae entre el valor más bajo y el más alto del valor crítico, la prueba no es conclusiva, y se requiere conocer el rango de cointegración para completar los cálculos. Los valores críticos para muestras finitas son proporcionados por Narayan (2005).

Además, Pesaran et al. (2001, pp. 8-9) ofrecen cinco interpretaciones alternativas del modelo corrección del error de la ecuación 3, distinguiéndose según si se le incorporan términos determinísticos - esto es intercepto y tendencia- Si estos se incorporan como parte del término de corrección del error, implícitamente se proyectan en el espacio del vector cointegrante, por lo tanto, los coeficientes $a_{0}$ y $a_{1}$ deben estar restringidos, es decir, sus coeficientes deben contrastarse en la hipótesis nula de la prueba Límite.

Los casos que consideran son:

Caso 1. Sin intercepto y sin tendencia: $a_{0}=0 ; a_{1}=0$.

Caso 2. Intercepto restringido y no tendencia: $a_{1}=0$. El intercepto entra en la ecuación cointegrante y su coeficiente se contrasta en la hipótesis nula de la prueba límite.

Caso 3. Intercepto sin restricción y no tendencia: $a_{0} \neq 0, a_{1}=0$. El intercepto se ignora en la ecuación de cointegración y no se evalúa el coeficiente en la hipótesis nula.

Caso 4. Intercepto sin restricción y tendencia restringida: $a_{0} \neq 0$. La tendencia se incorpora a la ecuación cointegrante y su coeficiente se contrasta en la hipótesis nula.

Caso 5. Intercepto sin restricción y tendencia sin restricción. $a_{0} \neq 0$, $a_{1} \neq 0$. La tendencia se ignora en la ecuación cointegrante, y su coeficiente no se evalúa en la hipótesis nula.

La medición de la respuesta de la oferta agraria a distintos factores con este enfoque econométrico ha sido utilizado por Bracamonte et al. (2018) en ganado vacuno vivo del departamento de Sucre, Colombia; Ogundari (2016) a la oferta y los precios del maíz en Nigeria; Maleki et al. (2012) a la oferta de exportación de carne en Irán; Van Wyk y Treurnicht (2012) a la oferta 
Castillo Núñez: Determinantes de la oferta primaria de ganado vacuno para ceba...

de carne de oveja; Ozkan y Karaman (2011) al área de algodón en Turquía; Muchapondwa (2009) a la oferta agregada de los cultivos en Zimbawe.

\section{A. Datos y fuentes}

Variable dependiente: $y_{t}=q l=$ oferta primaria de ganado vacuno vivo para ceba. Es la cantidad mensual vendida de ganado vacuno macho y hembra en pie de primera calidad entre 130 y 250 kilogramos de peso físico en subastas comerciales de Montería, Planeta Rica y Sahagún, Córdoba.

Variables explicativas: $x_{1}=p l=$ precio del ganado vacuno para ceba. Se formó como un promedio ponderado por las cantidades del precio por kilo de las edades consideradas y del sexo. Se mide en pesos colombianos corrientes por kilogramo de ganado vivo.

$x_{2}=r p=$ riesgo-precio. Es la desviación estándar del precio del ganado vacuno de levante en pie. Se calculó a partir de una media móvil del precio corriente por kilogramo retardado tres meses.

$x_{3}=r c=$ riesgo-clima. Es el coeficiente de variación de las precipitaciones pluviales mensuales en milímetros en 37 estaciones de Córdoba. Se construyó con base en información original sobre precipitaciones pluviales (lluvias) cuya fuente es el Instituto de Hidrología, Meteorología y Estudios Ambientales, IDEAM (s. f.).

$x_{4}=p l c=$ precio del coproducto. Es el precio pagado al productor de leche cruda de vaca en la unidad productiva por la industria lechera. La información entre enero/2007-septiembre/2012 es del Ministerio de Agricultura y Desarrollo Rural y de la Unidad Seguimiento de Precios de la leche, (MADR-USP, s. f.); corresponde al precio sin bonificación. Entre octubre/2012-diciembre/2018, se tomó el precio mensual reportado por el Sistema de información de precios del sector agrario, SIPSA, del DANE (DANE-SIPSA, s. f.), expresado en pesos colombianos corrientes por litro de leche cruda.

$x_{5}=p s h=$ Porcentaje de sacrificio de hembras de ganado vacuno en la región Caribe Colombiana. Se utiliza como variable proxy del ciclo de la ceba en la región. Se construyó con base en la información mensual 
de la Encuesta de sacrificio de ganado del DANE (DANE, 2018b), entre enero/2007-diciembre/2017. Enero/2018-diciembre/2018 es predicción del OPCA con base en la serie histórica del DANE.

$x_{6}=$ pru $=$ precio de la urea como proporción del precio del ganado vacuno cebado vivo de 3 años en Córdoba. Es una variable proxy al costo de producción.

$x_{7}=$ prm $=$ precio relativo del maíz amarillo con respecto al precio del ganado cebado vivo cebado en Córdoba. Es una aproximación a la rentabilidad de productos competentes en recursos.

\section{B. Procedencia de la información}

La información de oferta, precio del ganado, y demás variables proxy procede de las bases de datos del OPCA (2018), de la Universidad de Córdoba, Colombia. La periodicidad de las variables utilizadas es mensual. La variable $y_{t}$, y las variables independientes $x_{1}, x_{4}, x_{6} \mathrm{y} x_{7}$, fueron transformadas a logaritmo natural $(L)$, lo cual permite interpretarlos como elasticidades. Se usó el software Econometric -Views Versión 10 para estimación de los parámetros del modelo.

\section{Discusión de resultados}

\section{A. Descripción, características de los datos y estimación}

El comportamiento de la variable dependiente oferta de ganado vacuno vivo para ceba se muestra en la Figura 1. Su comportamiento deja ver la existencia de una tendencia decreciente desde los años 2012-2013, por lo que es probable que exija la inclusión de una tendencia determinística en la ecuación de oferta.

El número medio de cabezas mensuales destinadas a ceba fue 13181; el precio: $\$ 3305 /$ kilo; precio de leche cruda, $\$ 763 /$ litro; la desviación estándar de la media móvil del precio retardado 3 meses es $\$ 40,9$ por kilo; el coeficiente de variación de las lluvias, 0,77 ; el sacrificio de hembras respecto al sacrificio total, 0,41; el precio relativo de un kilo de urea, 0,34; un kilo de 
ganado cebado se intercambia en el mercado con 3,9 kilos de maíz amarillo. La distribución probabilística del precio de la leche y del precio relativo del maíz es la normal; para el resto se rechaza la hipótesis nula de acuerdo con el estadístico Jarque-Bera.

Figura 1. Evolución de la oferta de ganado vacuno de levante para ceba, enero/2007-diciembre/2018, (número de cabezas). Departamento de Córdoba

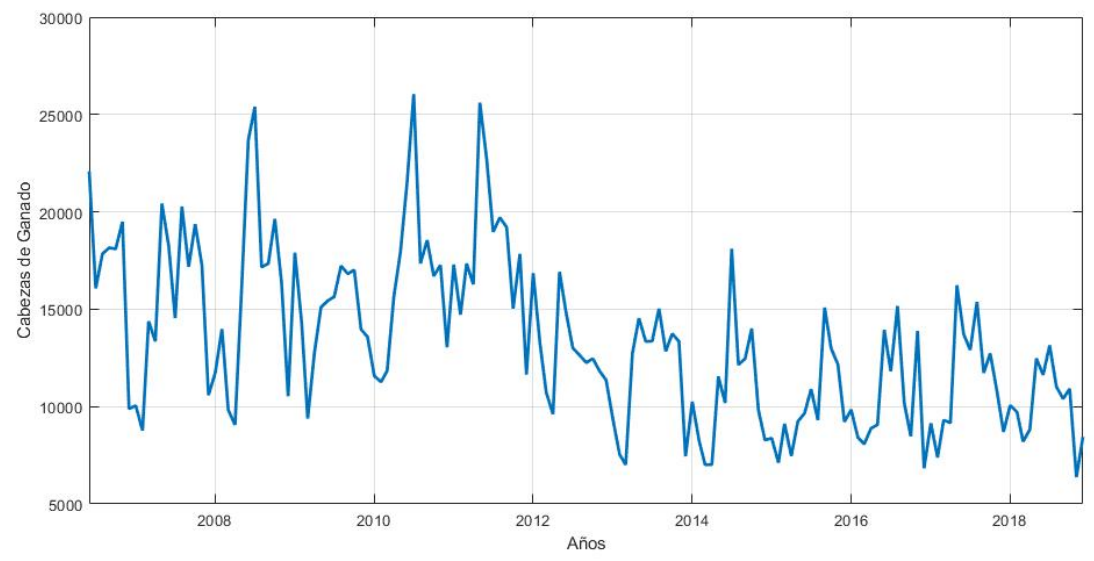

Fuente: OPCA (2018).

Para probar que las variables no son integradas de orden 2, se realizó la prueba de raíz unitaria de Zivot-Andrews (Zivot \& Andrews, 1992). Esta prueba toma en cuenta la existencia de cambio estructural en las variables, lo cual es una ventaja frente a las otras pruebas de raíz unitaria en las que no es considerado; el desconocimiento de cambio estructural reduce su poder sesgando a la aceptación de la hipótesis nula de existencia de una raíz unitaria (Perron, 1989).

En la Tabla 1 se muestran los resultados de la prueba. Ninguna de las variables consideradas es integrada de orden 2; lo son $I(0)$, es decir, estacionarias, e $I(1)$. En consecuencia, el enfoque ARDL puede ser aplicado. Las dos variables $I(1)$ muestran puntos de quiebre en noviembre/2008 y junio/2016, respectivamente; por ende, se introdujeron dos variables dummies que toman el valor de 1 en esas fechas de quiebre y 0 en las demás fechas. 
Tabla 1. Prueba de raíz unitaria de Zivot y Andrews sobre las variables de análisis. Modelo con intercepto y tendencia

\begin{tabular}{lccc}
\hline Variables & $\begin{array}{c}\text { Estadístico calculado } \\
\text { (v/bles en niveles) }\end{array}$ & $\begin{array}{c}\text { Estadístico calculado } \\
\text { (primeras diferencias) }\end{array}$ & $\begin{array}{c}\text { Orden de } \\
\text { integración }\end{array}$ \\
\hline$y_{t}$ oferta & $-7,00$ & & $I(0)$ \\
$x_{1}=$ precio & $-4,13$ & $-10,62$ & $I(1)$ \\
$x_{2}=$ riesgo-precio & $-8,73$ & & $I(0)$ \\
$x_{3}=$ riesgo-clima & $-10,46$ & & $I(0)$ \\
$x_{4}=$ precio leche cruda & $-6,33$ & $-13,64$ & $I(0)$ \\
$x_{5}=$ \% sacrificio hembras & $-4,53$ & & $I(1)$ \\
$x_{6}=$ gasto relativo urea-plag & $-5,67$ & $I(0)$ \\
$x_{7}=$ precio relativo maíz & $-5,63$ & $I(0)$ \\
\hline
\end{tabular}

Valor crítico al $1 \%=-5,34$; al $5 \%=-4,85$; al $10 \%=-4,60$

Fuente: elaboración propia.

Para contrastar el supuesto de no correlación de las perturbaciones de la ecuación 3, se estimó el vector autorregresivo, $\operatorname{VAR}(p)$, subyacente en el enfoque ARDL, con y sin tendencia determinística, con el fin de seleccionar apropiadamente el número de retardos de las variables, $p, \mathrm{y}$ corroborar la existencia de una tendencia determinística observable en la Figura 1. Hay un delicado balance en esta elección de modo que este debe ser lo suficientemente largo para evitar problemas de correlación serial y lo suficiente pequeño para que el modelo no quede sobre- parametrizado.

Evaluados con el criterio de información de Akaike, AIC, y el de Schwarz, SC, los resultados indican que, con la inclusión de una tendencia lineal determinística, el número de retardos óptimos del VAR son 3, y 1, respectivamente.

Con ese orden de retardos se acepta la hipótesis nula de no correlación serial del retardo 1 hasta 12. El valor mínimo que toman ambos criterios de información es ligeramente menor con la tendencia que sin ella. Este hecho y el comportamiento decreciente observado de la oferta en la Figura 1 
condujeron a una estimación de los casos 5 y 4 del modelo general, que contienen una tendencia.

La ecuación 5 es la representación más general del modelo.

$$
\begin{aligned}
\Delta y_{t}=a_{0}+a_{1} t+b_{0} y_{t-1}+ & \sum_{j=1}^{k} b_{j} x_{j, t-1}+\sum_{i=1}^{p-1} c_{0, i} \Delta y_{t-i}+ \\
& \sum_{j=1}^{k} \sum_{l_{j=1}}^{q_{j}-1} c_{j, l_{j}} \Delta x_{j, t-l_{j}}+\sum_{j=1}^{k} d_{j} \Delta x_{j, t}+\varepsilon_{t} .
\end{aligned}
$$

La prueba límite del caso 5 contrasta la hipótesis nula:

$$
\begin{aligned}
& H_{0}=b_{0}=b_{1}=b_{2}=b_{3}=b_{4}=b_{5}=b_{6}=b_{7}=0 \\
& H_{1}=b_{0}=b_{1}=b_{2}=b_{3}=b_{4}=b_{5}=b_{6}=b_{7} \neq 0
\end{aligned}
$$

El caso 4: $H_{0}=a_{1}=b_{0}=b_{1}=b_{2}=b_{3}=b_{4}=b_{5}=b_{6}=b_{7}=0$.

Con 3 retardos de la variable dependiente y 3 retardos de las variables independientes, sobre un total de 49152 modelos evaluados con el criterio de información AIC, el modelo óptimo seleccionado es el caso 5:

$$
\operatorname{ARDL}(p, q)=\operatorname{ARDL}(3,0,3,3,3,2,0,3) .
$$

Esto es, el número de retardos de la variable dependiente es 3 . El de las variables independientes, $x_{1}, \mathrm{y} x_{6}$ es $0 ; x_{2}, x_{3}, x_{4}, \mathrm{y} x_{7}$ es 3 ; el de $x_{5}$ son 2 retardos.

\section{B. Resultados}

\section{Relaciones de largo plazo}

Siguiendo la metodología propuesta, se prueba la existencia - $\mathrm{O}$ node una relación de largo plazo entre la oferta de ganado y las variables independientes a través de la prueba $\mathrm{F}$ límite. Los resultados se muestran en la Tabla 2. 
Tabla 2. Resultados de la prueba $F$ de Limite. $H o=$ no existe una relación de cointegración

\begin{tabular}{|c|c|c|c|c|c|c|c|}
\hline \multicolumn{5}{|c|}{ Prueba F de Límite: caso 5} & \multicolumn{3}{|c|}{ Prueba F de Límite: caso 4} \\
\hline Prueba Estadística & Valor & Significancia \% & $I(0)$ & $I(1)$ & Valor & $I(0)$ & $I(1)$ \\
\hline \multicolumn{8}{|c|}{ Distribución asintótica $n=1000$} \\
\hline \multirow[t]{4}{*}{ Estadístico F. $(k=7)$} & 4,88 & 10 & 2,38 & 3,45 & 4,35 & 2,22 & 3,17 \\
\hline & & 5 & 2,69 & 3,83 & & 2,5 & 3,5 \\
\hline & & 2,5 & 2,98 & 4,16 & & 2,76 & 3,81 \\
\hline & & 1 & 3,31 & 4,63 & & 3,07 & 4,23 \\
\hline \multicolumn{8}{|c|}{ Tamaño de muestra 141. Muestras finitas $n=80$} \\
\hline & & 10 & 2,5 & 3,63 & & 2,01 & 3,05 \\
\hline & & 5 & 2,88 & 4,11 & & 2,33 & 3,46 \\
\hline & & 1 & 3,73 & 5,16 & & 3,02 & 4,35 \\
\hline
\end{tabular}

Fuente: salida de software.

En ambos casos, utilizando la distribución asintótica, se puede rechazar al 1,0\% de significancia la hipótesis nula de no existencia de cointegración entre las variables, independiente que sean todas $I(0)$ o $I(1)$. Se acepta, por lo tanto, la hipótesis alternativa. Con la distribución para muestras finita, se rechaza también $H_{0}$ pero al $5 \%$ de significación.

Los parámetros de la ecuación cointegrante y el p-valor están contenidos en la Tabla 3.

Existe una relación de largo plazo entre la oferta y el precio, predicha por la teoría económica. En términos de elasticidad, con nivel de significancia del $1 \%$, una variación porcentual del 1,0\% en el precio produce en la oferta una variación en el mismo sentido del 1,9\%. Esta reacción de la oferta al precio, es decir, cuánto varía la oferta al cambio de precio, manteniendo los demás factores constantes, se le conoce en microeconomía como la respuesta de la oferta agraria. Este resultado en la oferta primaria de ganado vacuno confirma la robustez de esa relación revisada en la literatura por Mundlak (2001) y Chavas (2019) cuando se estima la oferta de productos individuales y se le incorporan variables de riesgo. 
Castillo Núñez: Determinantes de la oferta primaria de ganado vacuno para ceba...

Tabla 3. Relaciones de largo plazo entre la oferta de ganado vacuno para ceba y sus determinantes. Variable dependiente Lql. Departamento de Córdoba

\begin{tabular}{lcc|cc}
\hline \multicolumn{2}{c|}{ Caso 5} & \multicolumn{2}{c}{ Caso 4} \\
Variables independientes $x_{j}$ & Coeficientes $b_{j}$ & p-valor & Coeficientes $b_{j}$ & p-valor \\
\hline Lpl & 1,92 & 0,001 & 1,92 & 0,001 \\
rp & $-0,005$ & 0,008 & $-0,005$ & 0,008 \\
rc & $-0,117$ & 0,261 & $-0,087$ & 0,424 \\
Lple & $-1,132$ & 0,212 & $-1,050$ & 0,250 \\
psh & 1,943 & 0,051 & 1,943 & 0,051 \\
Lpru & $-0,527$ & 0,083 & $-0,527$ & 0,083 \\
Lprm & $-0,852$ & 0,019 & $-0,852$ & 0,019 \\
Tendencia & & & $-0,009$ & 0,003 \\
\hline
\end{tabular}

Fuente: elaboración propia.

La evidencia empírica de esta relación en Córdoba se encuentra en Castillo et al. (2015), cuando la función de oferta-precio parcial se obtiene de la función de costo total. Esa misma reacción positiva, con metodología de cointegración y corrección del error, en ganado para consumo, ha sido reportada por Kibara et al. (2016) en Kenia; Yoon \& Brown (2017) en Estados Unidos.

Los productores reaccionan negativamente a la variabilidad del precio del producto. Este resultado es plausible: los agentes forman su expectativa de precio asociada al patrón de lluvias dual de la región: una época de lluvia entre mayo y agosto con valores medios de precipitaciones entre $107 \mathrm{y}$ 209 milímetros ( $\mathrm{mm}$ ) y otra época seca entre diciembre-marzo con valores mínimos entre 16 y $68 \mathrm{~mm}$. Esto afecta el precio de forma distinta en ganadería bajo pastoreo. Perciben que la varianza del precio no es constante: al 1\% de significación, una variación en la desviación estándar de $\$ 82$ en el precio retardado tres meses, por encima o por debajo de su media, reduce la oferta en $0,01 \%$. Este hecho está en consonancia con lo que predice la teoría económica: que el riesgo-precio desestimula a los productores (Sandmo,1971). Con varianza condicional en el tiempo dentro de una estructura ARCH, Holt 
y Aradhyula (1990) encontraron en la oferta de carne de pollo en Estados Unidos una elasticidad de -0,03. Haile et al. (2016), usando datos de panel de varios países durante el período 1961-2010, encontraron también en el caso de los cultivos, que el coeficiente de las variables de riesgo-precio son estadística y económicamente significativas en los modelos de respuesta de la oferta de los cultivos de trigo, arroz, maíz y soya.

El cultivo del maíz y la oferta son productos competentes: una variación del $1 \%$ del precio relativo del maíz amarillo hace que la oferta varíe en sentido contrario en $0,85 \%$. Igual relación inversa encontró Hertford y Nores (1982) en la oferta final de ceba con el cultivo de algodón. El resultado es señal que el uso de cereales como alimento animal no es una opción técnica atractiva en regiones con relativa abundancia de tierra (Nin et al. 2007).

Existe una relación inversa entre la oferta y el costo relativo del manejo de praderas. Con un nivel de confianza del $90 \%$, una variación del $1 \%$ de este, produce una respuesta en sentido contrario de la oferta en $0,53 \%$. Aunque esta es una variable proxy, resalta que los ganaderos responden al precio relativo: si la variación del precio del producto final es superior a la del costo, esto es, disminuye el costo relativo, aumenta la oferta para ceba.

La variable proxy al ciclo de ceba tiene signo positivo: al $10 \%$ de significación, a una variación del $1 \%$ en el sacrificio de hembras, la oferta responde en la misma dirección afectándola en 1,9\%. El signo positivo de esta relación es consistente con las características de la ganadería de esta región:

i) La intensidad del ordeño del ganado en el sistema de producción de doble propósito ha modificado el mecanismo tradicional del ciclo en los agentes económicos. Para un productor que integra la producción de ceba, un aumento de la tasa de sacrificio por la disminución del precio del producto final no lo conduce necesariamente a liquidar ganado joven para sacrificio sino a comprar más para producir más del otro producto leche y vender después más crías. De hecho, según cálculos del OPCA (2018), durante 2007-2018 el precio relativo del litro de leche cruda de vaca con respecto al precio del kilo de ganado vivo cebado de 3 años pasó de 0,18 a 0,32; es decir, se valorizó. Bajo racionalidad, es de esperar incrementos de la oferta dentro de la unidad productiva 
Castillo Núñez: Determinantes de la oferta primaria de ganado vacuno para ceba...

(Suárez, 2005). Este resultado, sin embargo, no es observable con los datos de subastas, pero sí en un comercializador, agente predominante en éstas. Este buscará realizar un margen alto proveniente tanto de la diferencia entre el precio pagado por el producto en la unidad productiva y el precio de venta en subastas organizadas ${ }^{4}$, así como de la ceba intermedia que realiza.

ii) Un indicador — también proxy - al ciclo es la evolución del inventario ganadero, pero no existe información con periodicidad mensual. En Córdoba, la correlación entre las variaciones del inventario y las variaciones de la oferta de ganado de levante es positiva al $5 \%$ de significancia: un menor inventario, expresión de una fase de liquidación, está asociado a una menor oferta; un inventario mayor, expresión de una fase de retención, está asociado a una mayor oferta ${ }^{5}$.

Finalmente, el riesgo-clima tiene signo correcto, pero no es significativo en el largo plazo, lo cual la define como variable de corto plazo. El precio de la leche cruda de vaca no fue significativo, y tiene signo no esperado.

\section{Relaciones de corto plazo}

En esta sección del artículo se resalta las relaciones de corto plazo dadas por los coeficientes $c_{0, i}$ de los retardos de las variaciones de la oferta de ganado, los $c_{j, l_{j}}$ de los retardos de las variaciones de las variables independientes, y los $d_{j}$ de las variaciones de las variables independientes, que contiene la ecuación 5 . La incidencia empírica de estos se muestra en la Tabla 4.

El coeficiente de ajuste del error es alto $(-0,47)$ y tiene el signo correcto: indica que los desequilibrios se corrigen en 2 semanas (14 días).

4 Los cálculos del OPCA (2018) con base en la información de DANE (2018b) sobre sacrificio de ganado en el caribe colombiano indican que, en fase de sacrificio alto (enero/2007junio/2012), la correlación entre la tasa de sacrificio de hembras y las primeras diferencias del precio relativo tiene signo positivo, pero no es significativa. Igual asociación en las dos variables se tiene en fase de sacrificio bajo, julio/2012-diciembre/2018.

5 Según las Encuestas Nacionales Agropecuarias, ENA, del DANE (2018 a), el inventario de ganado vacuno en Córdoba, entre 1997-2000/2003 creció de 2,6 a 2,8 millones; con altibajos, se redujo desde el año 2004 hasta 2,0 millones en el año 2018. 
Los términos determinísticos, el intercepto y la tendencia, son significativos al $1 \%$. Esta última indica que, en promedio, la oferta decreció durante el periodo a un ritmo de $-0,004 \%$.

La variación de la oferta retardada del mes anterior y de dos meses atrás se relaciona de manera inversa con la variación de la oferta corriente: una variación del $1 \%$ del mes anterior provoca una reacción en sentido contrario del $0,33 \%$ y $0,17 \%$ — respectivamente- con nivel de significancia del 1 y $5 \%$.

La variación de la desviación estándar del precio del mes corriente y de dos meses atrás afectan de manera diferente la variación de la oferta actual: la primera la disminuye en $0,001 \%$, la del segundo mes la aumenta en $0,002 \%$, al $5 \%$ y $1 \%$ de significación, respectivamente.

El efecto del riesgo-clima es superior al de riesgo-precio. Una variación de las lluvias por encima o por debajo del promedio del mes corriente y de dos meses atrás provocan una variación de la oferta corriente en sentido contrario de $0,05 \%$ y $0,07 \%$, respectivamente.

La variación del precio de la leche cruda del mes actual y la de uno y dos meses atrás afecta en sentido inverso la variación de la oferta del mes corriente: un cambio positivo del $1 \%$, la disminuye en $1,1 \%$ y en $0,76 \%$, la del segundo mes en $0,81 \%$. Resultados en esa misma dirección con todas estas variables encontró Bracamonte, et al (2018); Jaramillo y Junguito (1993:62) en riesgoclima con cultivos a nivel nacional.

Con un nivel de confianza del $90 \%$, se comprueba que una variación del $1 \%$ un mes atrás en la tasa de sacrificio de hembras en la región, provoca en la oferta corriente una variación negativa del 1,05\%. En el corto plazo, existe un efecto indirecto del ciclo del cebado en la oferta del levante, no por vía de precio.

La variación del precio relativo del maíz amarillo retardado dos meses se relaciona de forma inversa con la variación de la oferta corriente: un aumento del precio relativo en $1 \%$ disminuye la variación de la oferta corriente de ganado en $0,48 \%$ con nivel de significación del $5 \%$. 
Castillo Núñez: Determinantes de la oferta primaria de ganado vacuno para ceba...

Tabla 4. Modelo Corrección del Error. Relaciones de corto plazo entre la oferta la oferta de ganado y sus determinates

\begin{tabular}{|c|c|c|c|c|}
\hline \multicolumn{5}{|l|}{ Variable dependiente: $\Delta L q l$} \\
\hline Variables Coeficientes & Error estándar & Estadístico & $\mathrm{t}$ & p-valor \\
\hline Coef. Aj. Error & $-0,470$ & 0,073 & $-6,441$ & $\mathrm{Nd}$ \\
\hline$a_{0}$ & 1,059 & 0,165 & 6,401 & 0 \\
\hline$a_{1}$ & $-0,004$ & 0 & $-5,769$ & 0,000 \\
\hline$\Delta L q l_{t-1}$ & $-0,327$ & 0,074 & $-4,404$ & 0,000 \\
\hline$\Delta L q l_{t-2}$ & $-0,175$ & 0,070 & $-2,510$ & 0,013 \\
\hline$\Delta r p$ & $-0,001$ & 0,001 & $-2,036$ & 0,044 \\
\hline$\Delta r p_{t-1}$ & $-0,001$ & 0,001 & 1,398 & 0,164 \\
\hline$\Delta r p_{t-2}$ & 0,002 & 0,000 & 3,868 & 0,000 \\
\hline$\Delta r c$ & $-0,053$ & 0,022 & $-2,382$ & 0,018 \\
\hline$\Delta r c_{t-1}$ & $-0,016$ & 0,026 & $-0,619$ & 0,537 \\
\hline$\Delta r c_{t-2}$ & $-0,073$ & 0,024 & $-3,046$ & 0,002 \\
\hline$\Delta L p l c$ & $-1,126$ & 0,413 & $-2,611$ & 0,010 \\
\hline$\Delta L p l c_{t-1}$ & $-0,760$ & 0,438 & $-1,736$ & 0,085 \\
\hline$\Delta L p l c_{t-2}$ & $-0,812$ & 0,419 & $-1,937$ & 0,055 \\
\hline$\Delta p s h$ & $-0,431$ & 0,633 & $-0,680$ & 0,497 \\
\hline$\Delta p s h_{t-1}$ & $-1,054$ & 0,629 & $-1,677$ & 0,096 \\
\hline$\Delta L p r m$ & $-0,226$ & 0,189 & $-1,197$ & 0,233 \\
\hline$\Delta \operatorname{Lprm}_{t-1}$ & 0,310 & 0,188 & 1,649 & 0,101 \\
\hline$\Delta \operatorname{Lprm}_{t-2}$ & $-0,481$ & 0,199 & $-2,414$ & 0,017 \\
\hline Du1108 & 0,273 & 0,199 & 1,370 & 0,173 \\
\hline$D u 0616$ & $-0,202$ & 0,297 & $-0,679$ & 0,498 \\
\hline
\end{tabular}

Fuente: elaboración propia. 


\section{Contrastación de los resultados}

El $R^{2}$ ajustado explica el $51,8 \%$ de las variaciones de la oferta. Los residuos poseen características estadísticas deseables: a) acepta al 5\% de significación la hipótesis nula de no correlación serial del estadístico F de la prueba $L M(12)$; b) son gaussianos, según la prueba de Jarque-Bera; c) son homocedásticos, evaluados según la prueba de Breuch-Pagan-Geoffrey. Contrastadas estas mismas características de los residuos a través del test especificación del error de la regresión, RESET (Ramsey, 1969), el estadístico F acepta la hipótesis nula $\varepsilon \sim N\left(0, \sigma^{2} I\right)$ al 2,5\% de significación ${ }^{6}$.

Las pruebas de estabilidad de los coeficientes fueron evaluadas a través de mínimos cuadrados recursivos, en particular la prueba CUSUM y CUSUM de cuadrados (Brown et al., 1975). Como se ve en Figura 2 -lado izquierdo-, los residuos recursivos divididos por la desviación estándar de los mismos, aunque se alejan de la línea cero, su salida se mantiene dentro de las líneas de significación del 5\%. En la Figura 2, lado derecho, la suma de cuadrados acumulativos permanece dentro de las líneas de significación del $5 \%$, sugiriendo que la varianza de los residuos es estable.

\section{Conclusiones}

Este artículo examinó los determinantes de la oferta de ganado vacuno para ceba en el departamento de Córdoba, Colombia, enero/2007diciembre/2018, utilizando la prueba límite del enfoque ARDL lineal. Los resultados aportan evidencia empírica adicional de la existencia de esas relaciones de largo y de corto plazo predichas por la teoría económica.

La oferta ganadera responde a estímulos de mercado. Reacciona positivamente al precio en el largo plazo; la respuesta a una variable proxy al costo relativo de producción se da en sentido inverso.

6 Se incorporó el cuadrado de los valores ajustados de las variables independientes de la regresión original. Por ende, se evaluó con 1 grado de libertad en el numerador y 112 en el denominador. 
Castillo Núñez: Determinantes de la oferta primaria de ganado vacuno para ceba...

Figura 2. Prueba CUSUM y CUSUM de cuadrados de estabilidad de los coeficientes del modelo


Fuente: elaboración propia.

Los ganaderos reaccionan al riesgo-precio y al riesgo-clima en sentido negativo. Esto es, responden con disminución de la oferta cuando la desviación estándar de estos dos factores se coloca por encima o por debajo de su media trimestral y mensual. Este resultado deja ver de nuevo problemas de volatilidad del ingreso económico y la importancia de idear índices de 
medición de riesgo, al igual que mecanismos de administración de este, sean públicos o privados.

La actividad ganadera y la del cultivo de maíz compiten por recursos productivos. En tal sentido, la política pública sobre mejorar la utilización del uso de los suelos se enfrenta a disyuntivas no solo técnicas - relacionadas con la aptitud agroecológica de éstos - sino también con cuestiones económicas relacionadas con precios y costos relativos. Refleja también que el uso de cereales como alimentación animal no es una opción técnica atractiva en regiones de relativa abundancia de tierras. Sin embargo, la combinación de cultivos con cría de animales es una expresión de agricultura multiproducto existente en la región.

Por la vía indirecta del precio relativo de la leche y del animal joven con respecto al producto final cebado, la oferta mantiene una relación positiva con el ciclo de la ceba a largo plazo. Esta respuesta es plausible por el fenómeno de intensificación del ordeño en el sistema de producción del doble propósito predominante en esta parte del país, el cual hace depender el ingreso económico ganadero no solo de la venta de animales sino también de la producción del coproducto leche. En el caso de los comercializadores - predominantes en los mercados organizados de subastas que, además, hacen actividad de ceba intermedia-, este resultado comprueba su objetivo de maximizar márgenes de comercialización inmediatas.

\section{Referencias}

Bracamonte, J., Arrieta, E., \& Castillo, O. (2018). Determinantes de la oferta de ganado vacuno para ceba en el departamento de Sucre, 2007 2014: Un enfoque ARDL. Revista de Economía del Caribe 21, 57-74. http:/www.scielo.org.co/pdf/ecoca/n21/2011-2106-ecoca-21-57.pdf

Brown, R., Durbin, J., \& Evans J. (1975). Techniques for Testing the Constancy of Regression Relationships over Time. Journal of the Royal Statistical Society, Series B, 37 (2), 149-192. http://www.jstor.org/stab le/2984889 
Castillo Núńez: Determinantes de la oferta primaria de ganado vacuno para ceba...

Cano, G. (2016). A manera de introducción: reflexiones e hipótesis sobre el sector agropecuario. En, G. Cano, A. Iregui, M. Ramírez \& A. Tribín (eds.), El desarrollo equitativo, competitivo y sostenible del sector agropecuario en Colombia (pp. 1-11). CAF-Banco de desarrollo de América Latina, Banco de la República.

Castillo, O. (2015). Economía agraria: Apuntes de clase. Fondo Editorial Universidad de Córdoba.

Castillo, O., Macea, M., \& Negrete, M. (2015). Microeconomía de la producción de ganado vacuno de carne en el valle medio del río Sinú, Montería-Colombia. Un estudio de caso. Revista Facultad de ciencias económicas. Investigación y reflexión, 23(2), 123-135. https://doi.org/10 $.18359 /$ rfce. 1611

Castillo, O. (2009). Mercados y precios del ganado en el noroccidente del Caribe Colombiano. Guadalupe.

Chavas, J. (2019): Role of Risk and Uncertainty in Agriculture. En G. Cramer, C. Paudel, \& A. Schmitz (eds), The Routledge Handbook of Agricultural Economics (pp. 603-615). Routledge.

Chavas, J. (2000). On information and Market Dynamics: The Case of The US Beef Market. Journal Economic Dynamics and Control, 24 (5-7), 833853. https://doi.org/10.1016/S0165-1889(99)00027-5

Chavas, J., Hummels, D., \& Wright, B. (2014). Introduction. En J. Chavas, D. Hummels \& B. Wright (eds), The Economics of Food Prices Volatility (pp. 1-11). University of Chicago Press.

Chavas, J., Chambers, R., \& Pope, R. D. (2010). A Century of Contributions Production Economics and Farm Management: A Century of Contributions. American Journal of Agricultural Economics, 92 (2), 356-375. https://www.jstor.org/stable/40647993?seq=1

Departamento Administrativo Nacional de Estadística (DANE). (2018a). Encuesta Nacional Agropecuaria (ENA). https://www.dane.gov.co/ index.php/estadisticas-por-tema/agropecuario/encuesta-nacional-a 
gropecuaria-ena/encuesta-nacional-agropecuaria-por-departamentos. Consultado el 12 de febrero de 2020.

Departamento Administrativo Nacional de Estadística (DANE). (2018b). Encuesta de sacrificio de ganado históricos. https://www.dane.gov.c $\mathrm{o} /$ index.php/estadisticas-por-tema/agropecuario/encuesta-de-sacri ficio-de-ganado/encuesta-de-sacrificio-de-ganado-esag-historicos. Consultado el 15 de junio de 2019.

DANE-SIPSA. (s. f.). Boletín mensual de precios de leche en finca. https: //www.dane.gov.co/index.php/estadisticas-por-tema/agropecuario /sistema-de-informacion-de-precios-sipsa/mayoristas-boletin-mensua 1-1/boletin-mensual-precios-de-leche-en-finca-historicos. Consultado el 20 de junio de 2020 .

Departamento Nacional de Planeación (DNP). (1980). La economía ganadera en Colombia. Revista de Planeación y Desarrollo, 12(3), 91141. https://colaboracion.dnp.gov.co/CDT/RevistaPD/1980/pd_vXI I_n3_1980_art.4.pdf

Engle, R. F., \& Granger, C. W. (1987). Cointegration and Error Correction: Representation, Estimation and Testing. Econometrica, 55(2), 251-276. https://www.jstor.org/stable/i332753

Escobar, S., \& Rojo, A. (2014). El ciclo de ganado en Colombia. Una caracterización desde las series de tiempo [tesis de maestría, Universidad EAFIT]. https://repository.eafit.edu.co/xmlui/bitstream/handle/107 84/7864/AndresEduardo_RojoZapata_Sebastian_EscobarPorras_201 5.pdf;jsessionid=F9EC23B119DE50FE16E36A078DC09697?seque nce $=2$

Gale, P., Drew, L., David, G., \& Wooldridge (2009). The Effect of Climate Change on The Occurrence and Prevalence of Livestock Disease in Great Britain: A Review. Journal of Applied Microbiology, 106 (5), 14091423. https://doi.org/10.1111/j.1365-2672.2008.04036.x

García, J. (1983). Las políticas económicas y el sector ganadero en Colombia: 1950-1977. Cuadernos de Historia Económica y Empresarial [Serie de 
Castillo Núñez: Determinantes de la oferta primaria de ganado vacuno para ceba...

documentos, No.19]. Banco de la República, sucursal Cartagena. https: //www.banrep.gov.co/sites/default/files/publicaciones/archivos/chee_1 9.pdf

González, J. \& Bonilla, E (2016). Aproximación al mercado de tierras en Colombia. Instituto de Estudios Urbanos. Universidad Nacional de Colombia.

Haile, M., Kalkuhl, M., \& von Braun, J. (2016). Worldwide Acreage and Yield Response to International Price Change and Volatility: A Dynamic Panel Data Analysis for Wheat, Rice Corn and Soybeans. In, M. Kalkuhl, J. von Braun, \& M. Torero (eds), Food Prices Volatility and Its Implications for Security and Policy (pp. 139-165). Springer.

Hertford, R., \& Nores, G. (1982). Caracterización del sector ganadero de Colombia 1953-1975. CIAT.

Holt, M. \& Aradhyula, S. (1990). Price Risk in Supply Equations: An Application of GARCH Time Series Models to the U.S. Broiler Market. Southern Economic Journal, 57 (1), 230-242. https://www.jstor.org/st able/1060492? seq=1

Howden, S., Crimp, S., \& Stokes, C. (2008). Climate Change and Australian Livestock Systems: Impact researchs and Policy Issues. Australian Journal of Experimental Agriculture, 48(7),780-788. https://doi.org/ 10.1071/EA08033

Hudson, D. (2007). Agricultural Markets and Prices. Blackwell Publishing.

Instituto de Hidrología , Meteorología y Estudios Ambientales, IDEAM (s. f.). Valores totales mensuales de precipitación. Sistema de Información Nacional Ambiental (SIAC).

Jaramillo, C. F., \& Caicedo, E. (1996). Caracterización del ciclo ganadero [documento de trabajo]. Fedesarrollo. https://www.repository.fedes arrollo.org.co/bitstream/handle/11445/1386/Repor_Mayo_1996_Jar amillo_y_Caicedo.pdf? sequence=2\&isAllowed $=y$ 
Jaramillo, C. F., \& Junguito, R. (1993). Crisis agropecuaria y politica macroeconómica [serie Debates de Coyuntura Económica, No. 29], 4667. Fedesarrollo.

Jarvis, L. (1974). Cattle as Capital Goods, Ranchers as Portfolio Managers: An Application to Argentine. Journal of Political Economy, 82(3), 489-520. https://doi.org/10.1086/260209

Johansen, S. (1995). Likelihood-Based Inference in Cointegrated Vector Autoregressive Models. Oxford University Press.

Kibara, M., George, R., \& Gerald, M. (2016). Estimation of Supply Response of Livestock Product: The Case of Kajiado District. Economics 5(1), 814. https://doi.org/10.11648/j.eco.20160501.12

Lorente, L. (1978). Producción de ganado de carne en Colombia. Banco Ganadero.

Lorente, L. (1986). La ganadería bovina en Colombia. En A. Machado (coordinador). Problemas agrarios colombianos (pp. 331-368). CEGASiglo XX1.

Mader, T., Frank, J., Harrington, G., Hahn, G., \& Nienaber, J. (2009). Potential Climate Change Effect on Warm Season Livestock Production in The Great Plains. Climate Change, 97(3-4), 525-541. https://doi.or g/10.1007/s10584-009-9615-1

Maleki, B. Avestisyan, Z., \& Paseban, F. (2012). Factors Affecting Iran's Animal Products Export: A Co-Integration Analysis. Journal of Agricultural Science and Technology, 14(6), 1195-1203. https://www.sid.ir/en/Jour nal/ViewPaper.aspx?ID=283703

Ministerio de Agricultura y Desarrollo Rural (MADR), \& Unidad de Seguimiento de Precios de leche (USP). (s. f.). Precio pagado al proveedor sin bonificaciones voluntarias departamental. http://uspleche.minagricu ltura.gov.co/precio-pagado-al-proveedor-sin-bonificaciones-voluntari as-departamental.html Consultado el 15 de abril de 2018. 
Castillo Núńez: Determinantes de la oferta primaria de ganado vacuno para ceba...

Moschini, G., \& Hennessy, D. (2001). Uncertainty, Risk Aversion, and Risk Management for Agricultural Producers. En B. Gardner \& G. Rausser (eds). Handbook of Agricultural Economics, 1A. Agricultural Production (pp. 87-153). Elsevier.

Muchapondwa, E. (2009): Supply Response of Zimbabwean Agriculture: 1970-1999. African Journal of Agricultural and Resource Economics, 3(1), 28-42. https://ageconsearch.umn.edu/record/56954

Mundlak, Y. (2001). Production and Supply. En B. Gardner \& G. Rausser (eds), Handbook of Agricultural Economics, 1A. Agricultural production (pp. 3-85). Elsevier.

Mundlak, Y., \& Huang, H. (1996). International Comparisons of Cattle Cycles, American Journal of Agricultural Economics, 78(4), 855-868. https://doi.org/10.2307/1243843

Narayan, P. K. (2005). The Saving and Investment Nexus for China: Evidence from Cointegration Tests. Applied Economics, 37(17), 1979-1990. http s://doi.org/10.1080/00036840500278103

Nelson, C. \& Plosser, C. (1982). Trend and random walks in economic time series. Journal of Monetary Economics, 10, 139-162. http://128.151.24 6.4/a425/jme82_NP.pdf

Nerlove, M., \& Bessler, D. (2001). Expectations, Information and Dynamics. In B. Gardner \& G. Rausser (eds). Handbook of Agricultural Economics, 1A. Agricultural production (pp. 156-206). Elsevier.

Nin, A., Ehui, S. \& Benin, S. (2007). Livestock Productivity in Developing Countries: An Assessment. En R. Evenson \& P. Pingali (eds), Handbook of Agricultural Economics (vol. 3, pp. 2461-2532). Elsevier.

Ogundari, K. (2016): Maize Supply Response to Price and Nonprice Determinants in Nigeria: Bounds Testing Approach. International transactions in operational research, 25(5), 1-15. https://doi.org/10.1 111/itor. 12284 
Observatorio de Precios y Costos Agrarios del Noroccidente del Caribe Colombiano (OPCA). (2018). Precios y cantidades mensuales de ganado bovino de primera clase en el Depto de Sucre, Colombia, subastas comerciales [Base de datos]. https://www.unicordoba.edu.co/wp-c ontent/uploads/2019/07/precios-y-cantidad-ganado-bovino-en-Sucre $-\mathrm{y}-\mathrm{C} \% \mathrm{C} 3 \% \mathrm{~B} 3$ rdoba.xlsx

Ozkan ,B., \& Karaman, S. (2011). Acreage Response for Cotton Regions in Turkey: An Application of The Bounds Testing Approach to Cointegration. New Medit, 10(2), 42-50. https://newmedit.iamb.it /2011/04/11/acreage-response-for-cotton-regions-in-turkey-an-appli cation-of-the-bounds-testing-approach-to-cointegration/

Park, J. (1992). Canonical Cointegration Regressions. Econometrica, 60(1), 119-143. https://doi.org/10.2307/2951679

Parra, T., \& Gómez, M. (2008). Libre comercio y la cadena de carne de res: ¿Una amenaza real? [tesis de maestría en Economía, Universidad Javeriana]. https://repository.javeriana.edu.co/handle/10554/9568

Pérez, G. (2004) Los ciclos ganaderos en Colombia, 1950-2001 [documentos de trabajo sobre economía regional, No. 46]. Banco de la República, sucursal Cartagena. https://www.banrep.gov.co/sites/default/files/publ icaciones/archivos/DTSER-46.pdf

Perfetti, J., Escobar, D., Castro, F., Cuervo, B., Rodríguez, M., \& Vargas, J. (2012). Costos de producción de doce productos agropecuarios [informe]. FEDESARROLLO. https://www.repository.fedesarrollo.org.co/handl e/11445/378

Perron, P. (1989). The Great Crash, The Oil Price Shock, and The Unit Root Hypothesis. Econometrica, 57 (6), 1361-1401. https://doi.org/10.230 7/1913712

Pesaran M. H., \& Shin, Y. (1999). An Autoregressive Distributed Lag Modelling Approach to Cointegration Analysis. En The Ragnar Frisch Centennial Symposium (371-413). Cambridge University Press. http: 
Castillo Núńez: Determinantes de la oferta primaria de ganado vacuno para ceba...

//citeseerx.ist.psu.edu/viewdoc/download?doi=10.1.1.153.3246 \&rep=rep1\&type=pdf

Pesaran, M. H, Shin,Y., \& Smith, R. (2001). Bounds Testing Approaches to The Analysis of Level Relationships. Journal of Applied Econometrics,16(3), 289-326. https://doi.org/10.1002/jae.616

Phillips, P., \& Ouliaris, S. (1990). Asymptotic Properties of Residual Based Tests for Cointegration. Econometrica, 58(1), 165-193. https://doi.org/ $10.2307 / 2938339$

Ramsey, J. B. (1969). Tests for Specification Errors in Classical Linear Least Squares Regression Analysis. Journal of the Royal Statistical Society, Series B, 31(2), 350-371. https://www.jstor.org/stable/2984219

Sandmo, A. (1971). On the Theory of the Competitive Firm Under Price Uncertainty. The American Economic Review, 61(1), 65-73. http://www. jstor.org/stable/1910541

Shin, Y., Yu, B., \& Greenwood-Nimmo, J. (2014). Modelling Asymmetric Cointegration and Dynamic Multipliers in a Nonlinear ARDL Framework. En W. Horrace \& R. Sickles (eds), Festschrift in Honor of Peter Schmidt (pp. 281-314). Springer Science and Business Media. https://doi.org/10.1007/978-1-4899-8008-3_9

Sistema de Información de Precios Agropecuarios (SIPSA). (2008). Boletín mensual precios de insumos y factores de producción pecuarios, 7(2), febrero. http://bibliotecadigital.agronet.gov.co/handle/11348/5720

Suárez, R. (2005). Incidencia de la academia y de los centros de investigación en las políticas ganaderas. En, A. Machado (coord.), La academia y el sector rural (tomo IV., pp. 17-67). Centro de Investigaciones para el Desarrollo- CID. Universidad Nacional de Colombia.

Tomek, W., \& Robinson, K. (2005). Agricultural Product Prices. Cornell University Press.

Tonsor, G., \& Mitchell, J. (2017): Evaluating Cattle Cycles: Changes over Time and Implications [working paper AM-GTT-2017.1]. Kansas State 
University, Department of Agricultural Economics. https://www.agma nager.info/sites/default/files/pdf/KSU_FactSheet_CattleCycle_01-31 -17.pdf

Van Wyk, D., \& Treurnicht, N. (2012). A Quantitative Analysis of Supply Response in the Namibian Mutton Industry. South African Journal of Industrial Engineering, 23(1), 202-215. https://doi.org/10.7166/23-1 $-231$

Yoon, J., \& Brown, S. (2017, 4-7 de febrero). Examination of Asymmetric Supply Response in the U.S. Livestock Industry [conferencia]. Annual Meeting, Agricultural Economics Association, Mobile, Alabama. https: //doi.org/10.22004/ag.econ.252779

Yver, R. (1972). Investment Behavior and the Supply Response of Cattle Farmers in Argentina [Ph.D. dissertation, University of Chicago].

Zivot, E., \& Andrews, D. (1992). Further Evidence on the Great Crash, the Oil-Price Shock, and the Unit-Root Hypothesis. Journal of Business \& Economic Statistics, 10, (3), 251-270. https://doi.org/10.2307/1391541 